\title{
The V16A polymorphism in SOD2 is associated with increased risk of diabetic nephropathy and cardiovascular disease in type 1 diabetes
}

\author{
A. Möllsten • A. Jorsal • M. Lajer • N. Vionnet • \\ L. Tarnow
}

Received: 12 June 2009 / Accepted: 14 September 2009 / Published online: 16 October 2009

(C) Springer-Verlag 2009

\begin{abstract}
Aims/hypothesis Hyperglycaemia increases oxidative stress and may thereby increase the risk of diabetic complications, including diabetic nephropathy. Cells are protected from oxidative damage by, for example, the manganese superoxide dismutase enzyme (MnSOD), but the functional polymorphism V16A affects the localisation of MnSOD and therefore its ability to scavenge superoxide radicals. In a Danish cohort of type 1 diabetes patients, we sought to confirm previous findings of association between the Vallele and the risk of diabetic nephropathy and to investigate the influence of this polymorphism on the development of cardiovascular disease.

Methods Type 1 diabetes patients attending the Steno Diabetes Center, Gentofte, Denmark, between 1993 and 2001 were enrolled in this study. A total of 441 cases with diabetic nephropathy (albumin excretion $\geq 300 \mathrm{mg} / 24 \mathrm{~h}$ ) and 314 controls with persistent normoalbuminuria $(<30 \mathrm{mg} / 24 \mathrm{~h})$, despite diabetes of duration $\geq 20$ years, were identified. The median duration of diabetes was 35 years (range 1273 years).

Results We confirmed the significant association between carrier status of the V allele and diabetic nephropathy. The association was independent of age at diabetes onset,
\end{abstract}

A. Möllsten $(\bowtie)$

Department of Clinical Sciences, Pediatrics, Umeå University, 90185 Umeå, Sweden

e-mail: anna.mollsten@pediatri.umu.se

A. Jorsal $\cdot$ M. Lajer $\cdot$ L. Tarnow

Steno Diabetes Center,

Gentofte, Denmark

N. Vionnet

INSERM and Université Pierre et Marie Curie-Paris, UMR S 525,

Paris, France
$\mathrm{HbA}_{1 \mathrm{c}}$, sex, smoking and diabetes duration (OR 1.7, 95\% CI 1.2-2.4). The VV and AV genotypes considered together also predicted the risk of cardiovascular disease, independently of age at follow-up, $\mathrm{HbA}_{1 \mathrm{c}}$, sex, smoking, systolic blood pressure, cholesterol and nephropathy status. The hazard ratio was 1.6 (95\% CI 1.0-2.5).

Conclusions/interpretation The MnSOD V16A polymorphism is involved in the development of nephropathy caused by type 1 diabetes and seems to predict cardiovascular disease during follow-up.

Keywords Cardiovascular disease - Diabetic nephropathy · MnSOD $\cdot$ SOD2 $\cdot$ Type 1 diabetes
Abbreviations
CVD Cardiovascular disease
ESRD End-stage renal disease
MnSOD Manganese superoxide dismutase

\section{Introduction}

The most important predictor of diabetic nephropathy is hyperglycaemia [1] and there is strong evidence that hyperglycaemia causes oxidative stress, which can influence multiple systems linked to diabetic complications [2]. The major source of intracellular reactive oxygen species is the mitochondrial respiratory chain. Manganese superoxide dismutase (MnSOD), encoded by the SOD2 gene, is translocated into the mitochondrial matrix, where it scavenges superoxide radicals to protect the cells from oxidative stress. In the targeting sequence of SOD2 there is a valine/ alanine polymorphism (rs4880 or V16A) [3]; valine (V) instead of alanine (A) results in less efficient transport of 
MnSOD into the mitochondrial matrix [4], which can compromise the ability to neutralise superoxide radicals.

In a previous study we found that the VV genotype was associated with increased risk of diabetic nephropathy in a group of Finnish and Swedish patients with type 1 diabetes [5]. In the present study we aimed to replicate our previous findings of association between the V16A polymorphism and diabetic nephropathy in a group of Danish patients with type 1 diabetes. Furthermore, we investigated whether this polymorphism predicted the development of cardiovascular disease (CVD) in patients with type 1 diabetes.

\section{Methods}

Study population From August 1993 until October 2001, all Danish type 1 diabetes patients of European extraction attending Steno Diabetes Center were invited to participate in a study included in the EURAGEDIC (European rational approach for the genetics of diabetic complications) project [6], which investigates genetic risk factors for the development of diabetic micro- and macrovascular complications. Patients with diabetic nephropathy had albumin excretion $\geq 300 \mathrm{mg} / 24 \mathrm{~h}$ in two out of three consecutive urine samples, presence of retinopathy and absence of other kidney diseases $(n=441)$. If retinopathy was not present, a kidney biopsy was required for the diagnosis of diabetic glomerulosclerosis. Among the cases with diabetic nephropathy, 99 had end-stage renal disease (ESRD), defined as being in need of dialysis or transplantation. Retrospective data on the onset of macroalbuminuria were obtained from patient records for the analysis of progression from macroalbuminuria to ESRD. Patients with persistent normoalbuminuria $(<30 \mathrm{mg} / 24 \mathrm{~h})$, despite at least 20 years duration of diabetes at follow-up $(n=314)$ and no treatment with antihypertensive medication, were included as controls. All patients had diabetes onset before 35 years of age and were insulin-dependent within 1 year from diagnosis. Information about smoking was based on standardised questionnaires. All patients gave their informed written consent. The study was performed in accordance with the Declaration of Helsinki and was approved by the regional ethics committee.

Follow-up Patients were followed until their last visit at Steno Diabetes Center or until 1 September 2006; median follow-up time was 8.7 years, range 0.0-12.9. Stroke, myocardial infarction, coronary artery bypass grafting and percutaneous coronary intervention were considered major cardiovascular events. Patients who experienced major CVD events before baseline were excluded from the calculations. Dates of non-fatal cardiovascular events were obtained from patient records or discharge letters from other hospitals. All death certificates were reviewed independently by two observers and the primary cause of death was recorded. The cause of death was classified as cardiovascular if no other cause of death was clearly established.

Genotyping and statistical methods Genotyping was performed as part of the EURAGEDIC study using standard methods as described recently [7]. In addition to the rs 4880 in exon 2, the rs2855116 in intron 3 and the rs5746136 in intron 5 were analysed. They were in linkage disequilibrium with rs4880 and the results were nearly identical; therefore, only the results for rs4880 are presented. Differences in clinical characteristics between groups were analysed using Pearson's $\chi^{2}$ test, the $t$ test and the MannWhitney $U$ test. Logistic regression and Cox regression analyses were used to calculate ORs and HRs and to adjust for possible confounding variables.

The study had $>80 \%$ power to detect association of the V16A with diabetic nephropathy at OR $\geq 1.6$ and with CVD at $\mathrm{HR} \geq 1.3$, calculated with the SPSS SamplePower program (SPSS, Chicago, IL, USA), assuming the genotype frequency to be 0.5 . SPSS 15.0 was used for the statistical calculations.

\section{Results}

Patients with diabetic nephropathy at baseline were younger at diabetes onset $(p<0.001)$ and had longer diabetes duration than the normoalbuminuric controls $(p<0.001)$. Patients with diabetic nephropathy had higher systolic and diastolic blood pressures and higher $\mathrm{HbA}_{1 \mathrm{c}}$ than the controls (comparisons at baseline, $p<0.001$ ). In the group of diabetic nephropathy patients, 125 had experienced at least one major CVD event and 82 had developed ESRD at follow-up (Table 1). Among the normoalbuminuric patients 29 had experienced at least one major CVD event (Table 1). There were almost as many normoalbuminuric patients as patients with diabetic nephropathy that reported ever smoking (Table 1). Ever smoking was significantly associated with CVD (OR 1.6, 95\% CI 1.1-2.4). When adjusting for age at follow-up, $\mathrm{HbA}_{1 \mathrm{c}}$ at baseline, sex, systolic blood pressure at baseline, cholesterol at baseline and nephropathy status, the increased risk was borderline significant (OR $1.6,95 \%$ CI $1.0-2.5$ ).

Genotype of rs4880 and diabetic nephropathy risk The rs4880 polymorphism was in Hardy-Weinberg equilibrium. In the normoalbuminuric controls the frequency of the $\mathrm{V}$ allele was 0.46 and in the diabetic nephropathy cases it was $0.53(p=0.01)$. The genotype frequencies for controls and cases were as follows: $\mathrm{AA}, 0.30$ and $0.25 ; \mathrm{AV}, 0.48$ and $0.50 ; \mathrm{VV}, 0.22$ and 0.26 , respectively $(p=0.034)$. Carriers 
Table 1 Clinical characteristics of type 1 diabetic patients with or without diabetic nephropathy, at baseline and follow-up

\begin{tabular}{|c|c|c|c|c|}
\hline \multirow[t]{2}{*}{ Characteristic } & \multicolumn{2}{|c|}{ Normoalbuminuria $(n=314)$} & \multicolumn{2}{|c|}{ Diabetic nephropathy $(n=441)$} \\
\hline & Baseline & Follow-up & Baseline & Follow-up \\
\hline Age (years) & $43.7 \pm 11.0$ & $52.3 \pm 10.9$ & $42.2 \pm 10.5$ & $51.2 \pm 10.1$ \\
\hline Age at diabetes onset (years) & $17.3 \pm 8.6$ & $17.3 \pm 8.6$ & $14.2 \pm 8.2$ & $14.2 \pm 8.2$ \\
\hline Duration of diabetes (years) & $23(15-63)$ & $34(22-68)$ & $27(7-65)$ & $36(12-73)$ \\
\hline $\mathrm{HbA}_{1 \mathrm{c}}(\%)$ & $8.3(5.7-11.5)$ & $7.8(5.2-11.6)$ & $9.3(5.4-14.0)$ & $8.5(4.6-14.2)$ \\
\hline $\mathrm{SBP}(\mathrm{mmHg})$ & $132 \pm 18$ & $136 \pm 16$ & $144 \pm 22$ & $141 \pm 20$ \\
\hline DBP $(\mathrm{mmHg})$ & $76 \pm 9$ & $76 \pm 9$ & $82 \pm 12$ & $76 \pm 11$ \\
\hline CVD, $n(\%)$ & $3(1.0)$ & $29(9.2)$ & $43(9.8)$ & $125(28.3)$ \\
\hline $\mathrm{ESRD}, n(\%)$ & - & - & $17(3.9)$ & $82(18.6)$ \\
\hline Urinary albumin excretion (mg/24h) & $6(1-30)$ & $7(0-29)$ & $598(3-14545)^{\mathrm{a}}$ & $325(2-8241)$ \\
\hline Total cholesterol (mmol/l) & $4.8 \pm 1.0$ & $4.7 \pm 0.8$ & $5.6 \pm 1.2$ & $4.9 \pm 1.3$ \\
\hline Retinopathy (\% no/SR/PR) & $39.8 / 38.9 / 21.3$ & $15.3 / 58.9 / 25.5$ & $1.6 / 30.2 / 68.3$ & $1.4 / 21.3 / 76.6$ \\
\hline $\operatorname{Sex}(\%$ men $)$ & 54.5 & 54.5 & 61.5 & 61.5 \\
\hline Ever smoking (\%) & 63.1 & NA & 66.3 & NA \\
\hline
\end{tabular}

Data are mean $\pm \mathrm{SD}$, median (range) or percentage

${ }^{a}$ Some patients with previous persistent macroalbuminuria had urinary albumin excretion values $<300 \mathrm{mg} / 24 \mathrm{~h}$ as a result of treatment with antihypertensive medication at the time of investigation

DBP, diastolic blood pressure; NA, not available; PR, proliferative retinopathy; SBP, systolic blood pressure; SR, simplex retinopathy

of the $\mathrm{V}$ allele were at increased risk of having diabetic nephropathy (Table 2), adjusted for age at diabetes onset $(p<0.001), \mathrm{HbA}_{1 \mathrm{c}}$ at baseline $(p<0.001)$, sex $(p=0.062)$, ever smoking $(p=0.325)$ and diabetes duration at follow-up $(p=0.099)$. When comparing the two homozygous genotypes (AA with VV), the risk of having diabetic nephropathy was doubled among patients with the VV genotype, with adjusted OR 2.0 (95\% CI 1.3-3.2) (Table 2). The VV and AV genotypes considered together did not predict progression from macroalbuminuria to ESRD; on the contrary these alleles seemed to be protective (HR 0.6, 95\% CI 0.4-0.9).
Genotype and CVD development In the analyses of CVD development we used the time from onset of diabetes until a major CVD event. Patients with a major CVD event before baseline were excluded. The VV and AV genotypes considered together were associated with increased risk of developing CVD compared with the AA genotype (Table 2). The analysis was adjusted for other well-known risk factors for CVD; age at follow up $(p<0.001), \mathrm{HbA}_{1 \mathrm{c}}$ at baseline ( $p=$ $0.010)$, sex $(p=0.218)$, ever smoking $(p=0.022)$, systolic blood pressure at baseline $(p<0.001)$, cholesterol at baseline $(p=0.002)$ and nephropathy status $(p=0.114)$.

Table 2 MnSOD genotype associated risks of diabetic nephropathy and cardiovascular disease

\begin{tabular}{llllll}
\hline Genotype & $\begin{array}{l}\text { Crude OR for } \\
\text { nephropathy }\end{array}$ & $p$ value & $\begin{array}{l}\text { Adjusted OR for } \\
\text { nephropathy }\end{array}$ & $p$ value & $\begin{array}{l}\text { Development } \\
\text { of CVD }^{\mathrm{b}}(\mathrm{HR})\end{array}$ \\
\hline $\mathrm{AA}$ & Reference & & Reference & Reference \\
$\mathrm{AV}$ & $1.4(1.0-2.0)$ & 0.063 & $1.5(1.0-2.3)$ & 0.036 & $1.7(1.1-2.6)$ \\
$\mathrm{VV}$ & $1.7(1.1-2.6)$ & 0.011 & $2.0(1.3-3.2)$ & 0.004 & $1.5(0.9-2.5)$ \\
$\mathrm{VV}+\mathrm{AV}$ & $1.5(1.1-2.1)$ & 0.018 & $1.7(1.2-2.4)$ & 0.008 & $1.6(1.03-2.5)$ \\
\hline
\end{tabular}

Data are OR or HR with $95 \%$ CI

Genotype AA was used as reference and compared with genotypes VV and AV separately and VV and AV grouped together Patients experiencing CVD before baseline were excluded

${ }^{\mathrm{a}}$ Logistic regression was used to adjust for age at diabetes onset, $\mathrm{HbA}_{1 \mathrm{c}}$ at baseline, sex, ever smoking and diabetes duration at follow-up

${ }^{\mathrm{b}}$ Cox regression using time from onset of diabetes until development of CVD adjusted for age at follow-up, $\mathrm{HbA}_{1 \mathrm{c}}$ at baseline, sex, ever smoking, systolic blood pressure at baseline, cholesterol at baseline and nephropathy status 


\section{Discussion}

The present study confirms the previous findings that the rs $4880 \mathrm{~V}$ allele is associated with increased risk of diabetic nephropathy $[5,8]$. The risk of having diabetic nephropathy was doubled for patients with the VV genotype compared with those with the AA genotype and the association was independent of well-known risk factors for diabetic nephropathy. Furthermore, we found that carriers of the rs $4880 \mathrm{~V}$ allele were at increased risk of developing CVD, independently of known risk factors and nephropathy status. This is in line with results from studies in nondiabetic populations which have shown that the $\mathrm{V}$ allele increases the risk of coronary artery disease and myocardial infarction [9].

Glucose induces several pathways that can contribute to the development of diabetic complications, including the activation of protein kinase $\mathrm{C}$ and nuclear factor $\mathrm{kB}$ (NF$\mathrm{KB})$ and the formation of advanced glycation end-products. Nishikawa et al. [2] demonstrated that when the levels of mitochondrial reactive oxygen species were normalised, these glucose-induced changes were prevented, which shows the importance of keeping reactive oxygen species at low levels, particularly for diabetic patients. One of the essential enzymes for this is the MnSOD, and since the $\mathrm{V}$ allele results in lower levels of MnSOD and decreased degradation of reactive oxygen species [4], patients carrying this allele are likely to have a higher burden of oxidative stress.

Smoking is known to increase oxidative stress and it is generally accepted as a risk factor for diabetic nephropathy [10]. In the present study there were no differences in reported smoking when comparing patients with and without diabetic nephropathy, but smoking was associated with CVD.

The patients with diabetic nephropathy in our study had persistent macroalbuminuria and the controls had persistent normoalbuminuria despite at least 20 years duration of diabetes and no antihypertensive medication. Even if the number of participants in our study was limited, the strict classification of cases and controls is a way to strengthen the data. We found that progression from diabetic nephropathy to ESRD was not associated with the VV and $\mathrm{AV}$ genotypes considered together, but since the risk of CVD was higher in these patients, independently of diabetic nephropathy, they may have increased risk of death from CVD before they develop ESRD. This could be a possible explanation of the seemingly contradictory finding that the rs $4880 \mathrm{~V}$ allele was associated with lower risk of ESRD. This also suggests that using ESRD as the outcome in casecontrol studies may cause a bias in the data, since we may observe an effect of surviving instead of increased risk of more severe disease progression.

We conclude that the $\mathrm{V}$ allele of the SOD2 rs4880 polymorphism increases the risk of diabetic nephropathy and predicts cardiovascular disease in patients with type 1 diabetes. Our findings support the hypothesis that impairment in the MnSOD enzyme, which is involved in the elimination of oxidative stress, increases the risk of diabetic nephropathy and diabetic macrovascular complications.

Acknowledgements The EURAGEDIC study was funded from the European Commission through contract QLG2-CT-2001-01669. This project was also supported by grants from the Swedish Research Council (project no. 07531) and the Västerbotten County Council. G. Dahlquist is gratefully acknowledged for valuable scientific advice.

Duality of interest The authors declare that there is no duality of interest associated with this manuscript.

\section{References}

1. Parving HH, Osterby R, Ritz E (2000) Diabetic nephropathy. In: Brenner BM (ed) Brenner and Rector's The kidney. Saunders, Philadelphia, pp 1731-1773

2. Nishikawa T, Edelstein D, Du XL et al (2000) Normalizing mitochondrial superoxide production blocks three pathways of hyperglycaemic damage. Nature 404:787-790

3. Shimoda-Matsubayashi S, Matsumine H, Kobayashi T, Nakagawa-Hattori Y, Shimizu Y, Mizuno Y (1996) Structural dimorphism in the mitochondrial targeting sequence in the human manganese superoxide dismutase gene. Biochem Biophys Res Commun 226:561-565

4. Sutton A, Khoury H, Prip-Buus C, Cepanec C, Pessayre D, Degoul F (2003) The Ala16Val genetic dimorphism modulates the import of human manganese superoxide dismutase into rat liver mitochondria. Pharmacogenetics 13:145-157

5. Möllsten A, Marklund SL, Wessman M et al (2007) A functional polymorphism in the manganese superoxide dismutase gene and diabetic nephropathy. Diabetes 56:265-269

6. Tarnow L, Groop PH, Hadjadj S et al (2008) European rational approach for the genetics of diabetic complications-EURAGEDIC: patient populations and strategy. Nephrol Dial Transplant 23:161-168

7. Vionnet N, Tregouet D, Kazeem G et al (2006) Analysis of 14 candidate genes for diabetic nephropathy on chromosome $3 \mathrm{q}$ in European populations: strongest evidence for association with a variant in the promoter region of the adiponectin gene. Diabetes 55:3166-3174

8. Nomiyama T, Tanaka Y, Piao L et al (2003) The polymorphism of manganese superoxide dismutase is associated with diabetic nephropathy in Japanese type 2 diabetic patients. J Hum Genet 48:138-141

9. Fujimoto H, Taguchi J, Imai Y et al (2008) Manganese superoxide dismutase polymorphism affects the oxidized low-density lipoprotein-induced apoptosis of macrophages and coronary artery disease. Eur Heart J 29:1267-1274

10. Scott LJ, Warram JH, Hanna LS, Laffel LM, Ryan L, Krolewski AS (2001) A nonlinear effect of hyperglycemia and current cigarette smoking are major determinants of the onset of microalbuminuria in type 1 diabetes. Diabetes 50:2842-2849 